\title{
Correlation functions of the degenerate relativistic electron gas with high density
}

\author{
Vavrukh M. ${ }^{1}$, Dzikovskyi D. ${ }^{1}$, Solovyan V. ${ }^{2}$, Tyshko N. ${ }^{1}$ \\ ${ }^{1}$ Ivan Franko National University of Lviv \\ 8 Kyrylo and Methodiy Str., 79005 Lviv, Ukraine \\ ${ }^{2}$ Institute for Condensed Matter Physics \\ of the National Academy of Sciences of Ukraine \\ 1 Svientsitskii str., 79011, Lviv, Ukraine
}

(Received 1 July 2016)

\begin{abstract}
The two- and three-particle correlation functions of degenerate relativistic model of homogeneous electron gas with Coulomb interactions at $T=0 \mathrm{~K}$ in the momentum-frequency presentation in the local field approximation are investigated. These functions are sufficient for a correct calculation of the equation of state for the electron-nuclear model with the densities that correspond to the degenerate dwarfs.
\end{abstract}

Keywords: reference system approach, $n$-particle correlation functions, the local field correction function.

2000 MSC: $82 \mathrm{~B} 10,82 \mathrm{D} 10$

UDC: $537.31 .311,53.01$

\section{Introduction}

The theory of degenerate dwarfs is based on the electron-nuclear model of matter with high density. This is due to the fact that studied objects have the masses of order of magnitude as the Sun but an average densities $10^{5} \mathrm{~g} / \mathrm{cm}^{3}$. In such conditions even the existence of the bounded electron states is impossible, so the electronic structure of degenerate dwarfs can be classified as simple metallic systems in which all electrons are collectivized. Due to the high density the Fermi momentum has the order $m_{0} c$ therefore the adequate model for the investigation of the characteristics of the degenerate dwarfs is the electron-nuclear model with relativistic degenerate electron subsystem.

The calculation of degenerate dwarf structures is based on the use of a state equation model, which in turn requires a prior calculation of $n$-particle correlation functions of electron subsystem. In the developed by S. Chandrasekhar theory of the internal structure of the cold dwarfs the equation of state of ideal degenerate electron gas in the paramagnetic phase at $T=0 K$ is used $[1,2]$. The general theory of the degenerate dwarf structure which could interpret all the diversity of their known properties must take into account many other factors, the most important of which is the incomplete degeneration, a variable chemical composition and Coulomb interactions.

The influence of the inter-particle interactions is among the least studied in the theory of degenerate dwarfs. For the first time it was mentioned by E. Salpeter, who made an approximate assessment contribution of interaction to the equation of state of an electron gas in the Wigner-Seitz approximation, Thomas-Fermi and the RPA (random phase approximation) [3].

In order to build the equation of the paramagnetic state of the electron-nuclear model [4] there exists generalized reference system approach developed in the papers $[5,6]$ for description the nonrelativistic electron liquid. The aim of our work is the calculation of two- and three-particle correlation functions of the relativistic electron liquid model in a local field approximation.

In the homogeneous electron-nuclear model two parameters are featured - the nuclear charge $z$ and the relativistic parameter $y=\hbar k_{F}\left(m_{0} c\right)^{-1}$ that are determined by the concentration of electrons 
$\left(k_{F}=\left(3 \pi^{2} N_{e} / V\right)^{1 / 3}\right)$. The contribution of the electron-nuclear interactions to the thermodynamic potential or free energy of the model is presented in the form of power series in terms of dimensionless non-ideality parameter $\xi=z e^{2} k_{F} E_{F}^{-1}$, where $E_{F}=\left\{\left(m_{0} c^{2}\right)^{2}+\hbar^{2} k_{F}^{2} c^{2}\right\}^{1 / 2}-m_{0} c^{2}$ is the electron energy at the Fermi level. In the case of the degenerate dwarfs $y \gtrsim 1$, then

$$
\xi=z \alpha_{0} y\left\{\left(1+y^{2}\right)^{1 / 2}-1\right\}^{-1}
$$

( $\alpha_{0}=e^{2} / \hbar c$ is the fine structure constant) is a small parameter, as $z \leqslant 26$. The electron-nuclear model at dwarfs densities is weakly non-ideal that allows us to limit the contributions only of the second and third orders of perturbation theory on the electron-nuclear interactions.

\section{The general relations}

As in a non-relativistic theory $[4,5]$, the $n$-particle correlation functions of the interacting relativistic electron gas in a momentum-frequency representation $\mu_{n}\left(x_{1}, \cdots, x_{n}\right)$ are defined by respective polarization functions $M_{n}\left(x_{1}, \cdots, x_{n}\right)$,

$$
\begin{gathered}
\mu_{2}(x,-x)=M_{2}(x,-x)\left\{1+\frac{V_{q}}{V} M_{2}(x,-x)\right\}^{-1}, \\
\mu_{n}\left(x_{1}, \cdots, x_{n}\right)=M_{n}\left(x_{1}, \cdots, x_{n}\right) \prod_{i=1}^{n}\left\{1+\frac{V_{q_{i}}}{V} M_{2}\left(x_{i},-x_{i}\right)\right\}^{-1}, \quad n \geqslant 3,
\end{gathered}
$$

where $V_{q}=4 \pi e^{2} / q^{2}$ is the Fourier transform of Coulomb potential, $V$ is the volume of system, $x \equiv(\mathbf{q}, \nu)$, where $\mathbf{q}$ is a wave vector, $\nu_{m}=2 m \pi \beta^{-1},(m=0 ; \pm 1 ; \pm 2 ; \cdots)$ is Bose-Matsubary frequency, $\beta=\left(k_{B} T\right)^{-1}$.

We used the local field approximation [6,7], where

$$
\begin{gathered}
M_{2}(x,-x)=\mu_{2}^{0}(x,-x)\left\{1-\frac{V_{q}}{V} \mu_{2}^{(0)}(x,-x) G(x)\right\}^{-1} \\
M_{n}\left(x_{1}, \cdots, x_{n}\right)=\mu_{n}^{0}\left(x_{1}, \cdots, x_{n}\right) \prod_{i=1}^{n}\left\{1-\frac{V_{q_{i}}}{V} \mu_{2}^{(0)}\left(x_{i},-x_{i}\right) G\left(x_{i}\right)\right\}^{-1} .
\end{gathered}
$$

Here $G(x)$ is the dynamic local field correction function and $\mu_{n}^{0}\left(x_{1}, \cdots, x_{n}\right)$ are the correlation functions of an ideal relativistic electron system (without interaction) taking into account only the effects of symmetry in many-fermion system. According to the formulas (2), (3)

$$
\begin{aligned}
\mu_{2}(x,-x) & =\mu_{2}^{0}(x,-x) \varepsilon^{-1}(x), \\
\mu_{n}\left(x_{1}, \cdots, x_{n}\right) & =\mu_{n}^{0}\left(x_{1}, \cdots, x_{n}\right) \prod_{i=1}^{n} \varepsilon^{-1}\left(x_{i}\right) .
\end{aligned}
$$

There appears the effective dielectric function

$$
\varepsilon(x)=1+\frac{V_{q}}{V} \mu_{2}^{0}(x,-x)[1-G(x)] .
$$

It is well-known from a non-relativistic electron-liquid theory [7] that for the weakly non-ideal model the local field correction function is the universal characteristic of the variables $x=(\mathbf{q}, \nu)$ which does not depend on any parameter:

$$
G_{i d}(x)=-\left(2 \beta V_{q}\right)^{-1}\left\{\mu_{2}^{0}(x,-x)\right\}^{-2} \sum_{x_{1}} V_{q_{1}} \mu_{4}^{0}\left(x,-x, x_{1},-x_{1}\right) .
$$


The correlation functions of an ideal model of relativistic electrons are expressed through the oneparticle Green's function of this model:

$$
\begin{aligned}
\mu_{2}^{0}(x,-x)= & -\beta^{-1} \sum_{\mathbf{k}, s} \sum_{\nu^{*}} G_{\mathbf{k}, s}\left(\nu^{*}\right) G_{\mathbf{k}+\mathbf{q}, s}\left(\nu^{*}+\nu\right), \\
\mu_{3}^{0}\left(x_{1}, x_{2}, x_{3}\right)= & 2 \beta^{-1} \sum_{\mathbf{k}, s} \sum_{\nu^{*}} G_{\mathbf{k}, s}\left(\nu^{*}\right) G_{\mathbf{k}+\mathbf{q}_{1}, s}\left(\nu^{*}+\nu_{1}\right) \\
& \times G_{\mathbf{k}-\mathbf{q}_{2}, s}\left(\nu^{*}-\nu_{2}\right) \delta_{\mathbf{q}_{1}+\mathbf{q}_{2}+\mathbf{q}_{3}, 0} \delta_{\nu_{1}+\nu_{2}+\nu_{3}, 0}, \\
\mu_{4}^{0}\left(x_{1},-x_{1}, x_{2},-x_{2}\right)= & \beta^{-1} \sum_{\mathbf{k}, s} \sum_{\nu^{*}} G_{\mathbf{k}, s}\left(\nu^{*}\right) G_{\mathbf{k}-\mathbf{q}_{1}, s}\left(\nu^{*}-\nu_{1}\right) \\
& \times \sum_{\sigma= \pm 1} G_{\mathbf{k}-\sigma \mathbf{q}_{2}, s}\left(\nu^{*}-\sigma \nu_{2}\right)\left\{2 G_{\mathbf{k}, s}\left(\nu^{*}\right)+G_{\mathbf{k}+\mathbf{q}_{1}+\sigma \mathbf{q}_{2}, s}\left(\nu^{*}+\nu_{1}-\sigma \nu_{2}\right)\right\}
\end{aligned}
$$

etc., where

$$
G_{\mathbf{k}, s}\left(\nu^{*}\right)=\left\{i \nu^{*}-E_{k}+\mu\right\}^{-1} e^{i \delta \nu^{*}},
$$

$\delta \rightarrow+0, E_{k}=\left\{\left(m_{0} c^{2}\right)^{2}+\hbar^{2} k^{2} c^{2}\right\}^{1 / 2}-m_{0} c^{2}, \mu$ is the chemical potential variable. In the formulas (7) $\nu_{m}^{*}=(2 m+1) \pi \beta^{-1}$ is the Fermi-Matsubara frequency. Expanding the products of Green's functions into simple factors and performing a summation over frequency under the rule

$$
\beta^{-1} \sum_{\nu^{*}} G_{\mathbf{k}, s}\left(\nu_{*}\right)=n_{\mathbf{k}, s}=\left\{1+\exp \left[\beta\left(E_{k}-\mu\right)\right]\right\}^{-1},
$$

we obtain the next representation of the functions (7):

$$
\begin{aligned}
\mu_{2}^{0}(x,-x) & =-2 \operatorname{Re} \sum_{\mathbf{k}, s} n_{\mathbf{k}, s}\left\{i \nu+E_{\mathbf{k}}-E_{\mathbf{k}+\mathbf{q}}\right\}^{-1} \\
\mu_{3}^{0}\left(x_{1}, x_{2}, x_{3}\right) & =\delta_{x_{1}+x_{2}+x_{3}, 0}\left\{\gamma_{3}\left(x_{1},-x_{2}\right)+\gamma_{3}\left(x_{2},-x_{3}\right)+\gamma_{3}\left(x_{3},-x_{1}\right)\right\} \\
\gamma_{3}\left(x_{l},-x_{j}\right) & =2 \operatorname{Re} \sum_{\mathbf{k}, s} n_{\mathbf{k}, s}\left\{i \nu_{l}+E_{\mathbf{k}}-E_{\mathbf{k}+\mathbf{q}_{l}}\right\}^{-1}\left\{-i \nu_{j}+E_{\mathbf{k}}-E_{\mathbf{k}+\mathbf{q}_{j}}\right\}^{-1} .
\end{aligned}
$$

In the non-relativistic theory (for $E_{k}=\hbar^{2} k^{2} / 2 m$ ) the functions $\mu_{n}^{0}\left(x_{1}, \cdots, x_{n}\right.$ ) at $T=0 K$ are well known: $\mu_{2}^{0}(x,-x)$ is a polarization function which appears in RPA [8]; the static limits of functions (10) with $n \geqslant 3$ were investigated in $[9,10]$ and the dynamic functions $\mu_{n}^{0}\left(x_{1}, \cdots, x_{n}\right)$ with $n=3$ and 4 were calculated for the first time in the paper [4]. The direct calculation of the static and dynamic functions $\mu_{n}^{0}\left(x_{1}, \cdots, x_{n}\right)$ with $n \geqslant 3$ requires the integration all with respect to the vector $\mathbf{k}$ at the given vectors $\left(\mathbf{q}_{1}, \mathbf{q}_{2}, \cdots, \mathbf{q}_{n}\right)$ and is cumbersome. This difficulty can be overcome by using the Feynman identity [11]

$$
\prod_{j=1}^{n} B_{j}^{-1}=(n-1) ! \int_{0}^{1} \cdots \int_{0}^{1} d \alpha_{1} \cdots d \alpha_{n}\left\{\sum_{j=1}^{n} \alpha_{j} B_{j}\right\}^{-n} \delta\left(\sum_{j=1}^{n} \alpha_{j}-1\right),
$$

where $B_{j}=i \nu_{j}+E_{\mathbf{k}}-E_{\mathbf{k}-\mathbf{q}_{j}}$, making it easy to integrate with respect to angular variables of the vector $\mathbf{k}$.

The essential mathematical difficulty on the way of exact analytical calculation of functions $\mu_{n}^{0}\left(x_{1}, \cdots, x_{n}\right)$ with $n \geqslant 3$ in the relativistic case is that the identity (11) is not applicable in this case because of the strong dependence of $E_{\mathbf{k}}$ on a wave vector. 


\section{The two-particle correlation function}

In the case $n=2$, the integration with respect to the angular variables of vector $\mathbf{k}$ does not cause any difficulties and $\mu_{2}^{0}(x,-x)$ is reduced to the one-dimensional integral:

$$
\begin{aligned}
\mu_{2}^{0}(x,-x) & =3 N_{e}\left[m_{0} c^{2} y^{2}\right]^{-1}(2 q)^{-1} \int_{0}^{\infty} A(k|q, \bar{\nu}| y) n_{\mathbf{k}, s} k d k \\
A(k|q, \bar{\nu}| y) & =\eta_{+}-\eta_{-}+\frac{1}{2}\left(1+k^{2}\right)^{1 / 2} \ln \left[\frac{\bar{\nu}^{2}+\eta_{+}^{2}}{\bar{\nu}^{2}+\eta_{-}^{2}}\right]-\bar{\nu}\left\{\operatorname{arctg} \frac{\eta_{+}}{\bar{\nu}}-\operatorname{arctg} \frac{\eta_{-}}{\bar{\nu}}\right\} \\
\eta_{ \pm} & =\left\{1+y^{2}(k \pm q)\right\}^{1 / 2}-\left\{1+y^{2} k^{2}\right\}^{1 / 2}
\end{aligned}
$$

where "non-relativistic" variables $k \equiv|\mathbf{k}| k_{F}^{-1}, q \equiv|\mathbf{q}| k_{F}^{-1}, \bar{\nu}=\nu\left(m_{0} c^{2}\right)^{-1}=\tilde{\nu} y^{2} ; \tilde{\nu}=\nu\left(2 \varepsilon_{F}\right)^{-1}$, $\varepsilon_{F}=\hbar^{2} k_{F}^{2}\left(2 m_{0}\right)^{-1}$ are used.

At the absolute zero temperature (when $\left.\mu=E_{F}\right)$ in the static case $(\nu=0)$, the integration with respect to variable $k$ gives the following result:

$$
\begin{aligned}
\mu_{2}^{0}(\mathbf{q},-\mathbf{q})= & \lim _{\nu \rightarrow 0} \mu_{2}^{0}(x,-x)=\frac{3 N_{e}}{m_{0} c^{2} y^{2}} J_{2}\left(q_{*} \mid y\right), \\
q_{*} y J_{2}\left(q_{*} \mid y\right)= & \frac{2}{9}\left(R_{+}-R_{-}\right)\left[1+\frac{7}{4} y^{2}-\frac{q_{*}^{2}}{8}\right]+\frac{5}{72} q_{*} y\left(R_{+}+R_{-}\right)+\frac{q_{*} y}{12} R_{0}+\frac{1}{3} R_{0}^{3} \ln \left|\frac{R_{+}-R_{0}}{R_{-}-R_{0}}\right| \\
& +\frac{1}{8} q_{*}\left(1+\frac{q_{*}^{2}}{6}\right)\left\{2 \ln \left|y+R_{0}\right|-\ln \left|\left(R_{+}+y+q_{*}\right)\left(R_{-}+y-q_{*}\right)\right|\right\} \\
& +\frac{1}{6} S_{q}^{3}\left\{\ln \left|\frac{1+\frac{1}{2} q_{*}^{2}+\frac{1}{2} y q_{*}+S_{q} R_{+}}{1+\frac{1}{2} q_{*}^{2}-\frac{1}{2} y q_{*}+S_{q} R_{-}}\right|-\ln \left|\frac{1+\frac{1}{2} q_{*} y+S_{q} R_{0}}{1-\frac{1}{2} q_{*} y+S_{q} R_{0}}\right|-2 \ln \left|\frac{y+\frac{1}{2} q_{*}}{y-\frac{1}{2} q_{*}}\right|\right\} .
\end{aligned}
$$

Here the following notations are used:

$$
q_{*} \equiv y|\mathbf{q}| k_{F}^{-1} ; \quad R_{0}=\left(1+y^{2}\right)^{1 / 2} ; \quad S_{q}=\left(1+\frac{1}{4} q_{*}^{2}\right)^{1 / 2} ; \quad R_{ \pm}=\left[1+\left(q_{*} \pm y\right)^{2}\right]^{1 / 2} .
$$

This expression is an exact, unlike approximate representation from the paper [12]. The function $\mu_{2}^{0}(\mathbf{q},-\mathbf{q})$ has the following asymptotic:

$$
\mu_{2}^{0}(\mathbf{q},-\mathbf{q}) \Rightarrow \begin{cases}3 N_{e}\left(m_{0} c^{2} y^{2}\right)^{-1}\left(1+y^{2}\right)^{1 / 2}+\cdots & \text { by } q \ll k_{F} \\ 2 N_{e}(\hbar c q)^{-1}+\cdots & \text { by } q \gg k_{F}\end{cases}
$$

which differs from the non-relativistic function asymptotic

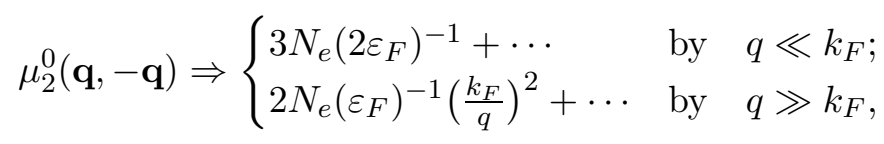

The dependence of function $J_{2}\left(q_{*} \mid y\right)$ on the relativistic parameter and wave vector (in units of $\left.q=|\mathbf{q}| / k_{F}\right)$ is illustrated in Fig. 1. The dynamic function $\mu_{2}^{0}(x,-x)$ that needs only to calculate the correlation energy model electron liquid can be used in the form (12) and can be calculated numerically, placing at $T=0 K n_{\mathbf{k}, s}=1$ for $k \leqslant 1, n_{\mathbf{k}, s}=0$ for $k>1$. Its dependence on the wave vector and relativistic parameter is illustrated in Fig. 2. However to identify common characteristics of this function is useful to obtain an approximate analytical expression. Based on the formula (10) through the identical transformation, it will be presented in the following form: 


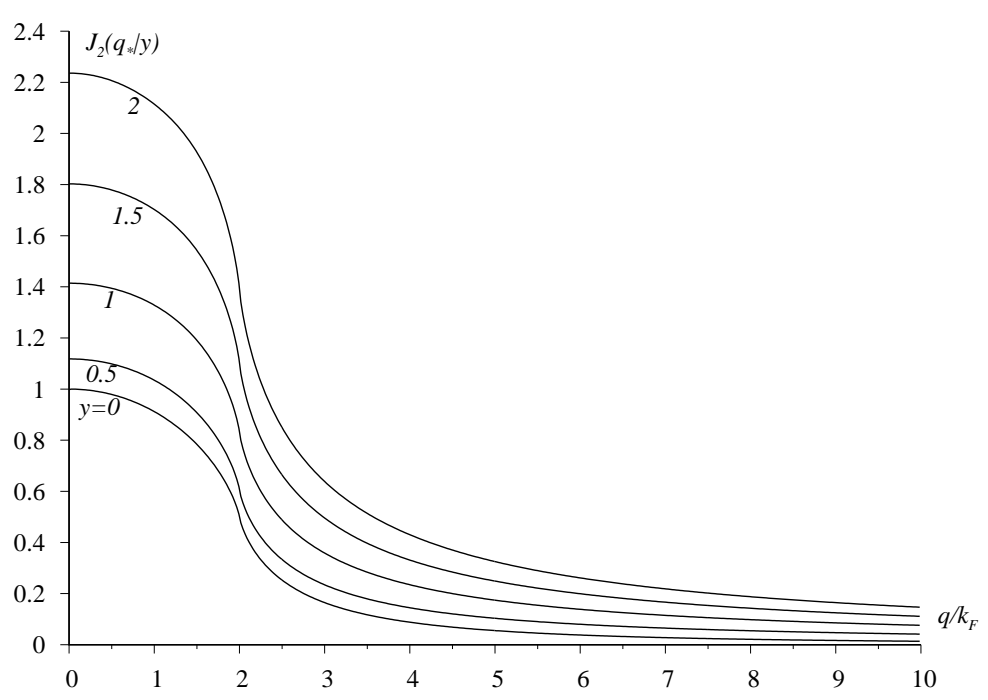

Fig. 1. Dependence of the function $J_{2}\left(q_{*} \mid y\right)$ on the wave vector $\mathbf{q}$ for different values of relativistic parameter.

$$
\begin{aligned}
\mu_{2}^{0}(x,-x) & =2 \sum_{\mathbf{k}, s} n_{\mathbf{k}, s}\left(\tilde{E}_{\mathbf{k}+\mathbf{q}}+\tilde{E}_{\mathbf{k}}\right)\left(\tilde{E}_{\mathbf{k}+\mathbf{q}}^{2}-\tilde{E}_{\mathbf{k}}^{2}\right)\left\{\left(\tilde{E}_{\mathbf{k}+\mathbf{q}}^{2}-\tilde{E}_{\mathbf{k}}^{2}\right)^{2}+\nu^{2}\left(\tilde{E}_{\mathbf{k}+\mathbf{q}}+\tilde{E}_{\mathbf{k}}\right)^{2}\right\}^{-1} \\
& =\frac{3 N_{e}}{m_{0} c^{2} y^{2}} \cdot \frac{1}{q} \int_{-1}^{1} d t \int_{0}^{1} \frac{d k k\left(t+\frac{q}{2 k}\right) C(k, t \mid q)}{\left(t+\frac{q}{2 k}\right)^{2}+\left(\frac{\nu}{2 k q} C(k, t \mid q)\right)^{2}}, \\
C(k, t \mid q) & =\frac{1}{2}\left\{\left[1+y^{2}\left(k^{2}+q^{2}+2 k q t\right)\right]^{1 / 2}+\left[1+y^{2} k^{2}\right]^{1 / 2}\right\}, \quad \tilde{E}_{\mathbf{k}}=\left\{\left(m_{0} c^{2}\right)^{2}+\hbar^{2} k^{2} c^{2}\right\}^{1 / 2} .
\end{aligned}
$$

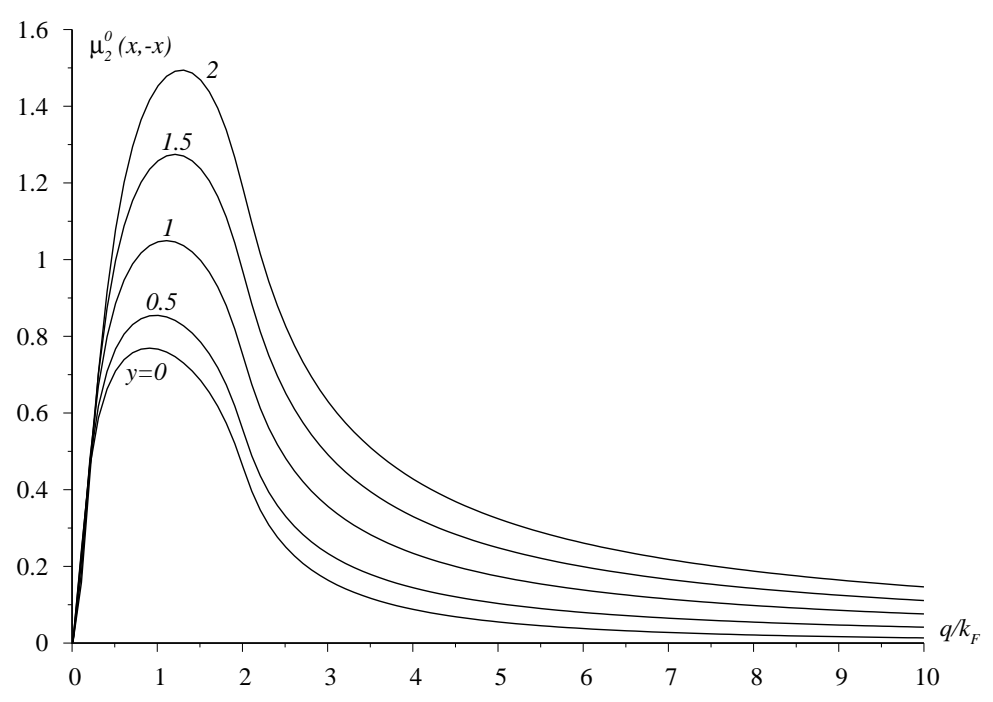

Fig. 2. Dependence of the dynamic function $\mu_{2}^{0}(x,-x)$ on the wave vector $\mathbf{q}$ for different values of relativistic parameter $\left(\nu=0.1 m_{0} c^{2} y^{2}\right)$.

To obtain an approximate analytical formula, a substitution must be performed

$$
C(k, t \mid q) \Rightarrow\left(1+y^{2}\right)^{1 / 2} ; \quad k^{-1} C(k, t \mid q) \Rightarrow y^{-1}\left(1+y^{2}\right)^{1 / 2} .
$$


As a result of the integration we obtain the following expression

$$
\begin{aligned}
\mu_{2}^{0}(x,-x)= & \frac{3 N_{e}}{m_{0} c^{2} y^{2}} \tilde{J}_{2}(q, v \mid y), \\
\tilde{J}_{2}(q, v \mid y) \approx & \frac{1}{2}\left(1+y^{2}\right)^{1 / 2}\left\{1+\frac{1}{2 q}\left(1+v^{2}-\frac{q^{2}}{4 y^{2}}\right) \ln \frac{v^{2}+(1+q / 2)^{2}}{v^{2}+(1-q / 2)^{2}}\right. \\
& \left.-v\left[\arctan \frac{1+q / 2}{v}+\arctan \frac{1-q / 2}{v}\right]\right\} .
\end{aligned}
$$

The difference of this expression from the non-relativistic case is the factor $\left(1+y^{2}\right)^{1 / 2}$ and frequency renormalization

$$
v=\frac{\nu}{2 \varepsilon_{F} q}\left(1+y^{2}\right)^{1 / 2}=\frac{\nu}{m_{0} c^{2} y^{2} q}\left(1+y^{2}\right)^{1 / 2} .
$$

It is calculated numerically due to the formula (12) and has a little deviation from the "exact" only in the vicinity of the maximum.

\section{The static three-particle correlation function}

In the particular case when $\mathbf{q}_{1}+\mathbf{q}_{2}=0$, this function is calculated exactly at the absolute zero temperature because

$$
\begin{aligned}
\mu_{3}^{0}(\mathbf{q},-\mathbf{q}, 0) & =\beta^{-1} \sum_{\mathbf{k}, s, \nu^{*}} G_{\mathbf{k}, s}\left(\nu^{*}\right) G_{\mathbf{k}+\mathbf{q}, s}\left(\nu^{*}\right)\left\{G_{\mathbf{k}, s}\left(\nu^{*}\right)+G_{\mathbf{k}+\mathbf{q}, s}\left(\nu^{*}\right)\right\} \\
& =\frac{d}{d \mu} \mu_{2}^{0}(\mathbf{q},-\mathbf{q})=2 \sum_{k, s}\left(E_{\mathbf{k}}-E_{\mathbf{k}+\mathbf{q}}\right)^{-1} \frac{d n_{\mathbf{k}, s}}{d \mu} .
\end{aligned}
$$

The equality $\frac{d}{d \mu} n_{\mathbf{k}, s}=\delta\left(E_{\mathbf{k}}-\mu\right)$ allows us to integrate with respect to the vector $\mathbf{k}$ and as a result we find that

$$
\begin{aligned}
\mu_{3}^{0}(\mathbf{q},-\mathbf{q}, 0) & =\frac{3 N_{e}}{\left(m_{0} c^{2} y^{2}\right)^{2}} J_{3}(q \mid y), \\
J_{3}(q \mid y) & =q^{-1} R_{0}\left\{R_{+}-R_{-}+R_{0} \ln \left|\frac{R_{+}-R_{0}}{R_{-}-R_{0}}\right|\right\},
\end{aligned}
$$

where $R_{ \pm}, R_{0}$ are defined by formulas $(14)$ and $q=|\mathbf{q}| / k_{F}$. In the non-relativistic limit $(y \ll 1)$

$$
\mu_{3}^{0}(\mathbf{q},-\mathbf{q}, 0)=\frac{3 N_{e}}{\left(m_{0} c^{2} y^{2}\right)^{2}} q^{-1} \ln \left|\frac{1+\frac{1}{2} q}{1-\frac{1}{2} q}\right| .
$$

The function (21) has long-wave asymptotic

$$
\frac{3 N_{e}}{\left(m_{0} c^{2} y^{2}\right)^{2}}\left(1+2 y^{2}\right)
$$

which differs by the factor $\left(1+2 y^{2}\right)$ from the asymptotic behavior of the function (21). The dependence of the dimensionless factor $J_{3}(q \mid y)$ on the wave vector and relativistic parameter is illustrated in Fig. 3. As one can see from this figure, in the wide domain of the vector $\mathbf{q}$ variation, the next relationship takes place

$$
\frac{J_{3}\left(q \mid y_{1}\right)}{J_{3}\left(q \mid y_{2}\right)} \approx \frac{1+2 y_{1}^{2}}{1+2 y_{2}^{2}}
$$




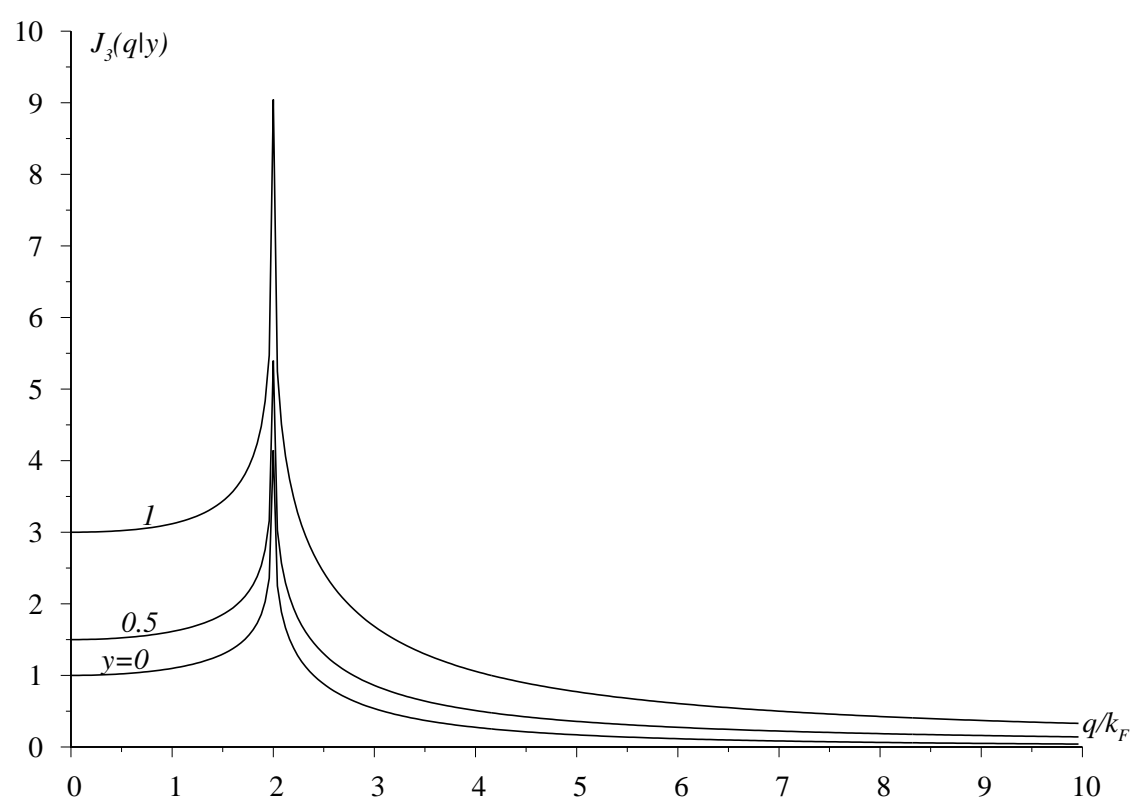

Fig. 3. Dependence of the static function $J_{3}(q \mid y)$ on the wave vector $\mathbf{q}$ for different values of relativistic parameter.

The short-wave asymptotic behavior of the function $(21)\left(\sim q^{-1}\right)$ differs from the non-relativistic asymptotic of the expression $(22)\left(\sim q^{-2}\right)$.

Due to the fact that in the domain of parameters, which corresponds to degenerate dwarfs, the three-particle correlations play a role of only correction, we calculate the function $\mu_{3}^{0}\left(\mathbf{q}_{1}, \mathbf{q}_{2},-\mathbf{q}_{1}-\mathbf{q}_{2}\right)$ approximately. Using the identity transformation, we present $\gamma_{3}\left(\mathbf{q}_{i}, \mathbf{q}_{j}\right)$ in the form

$$
\begin{aligned}
\gamma_{3}\left(\mathbf{q}_{i}, \mathbf{q}_{j}\right) & =2 \sum_{\mathbf{k}, s} n_{\mathbf{k}, s}\left\{E_{\mathbf{k}+\mathbf{q}_{i}}^{2}-E_{\mathbf{k}}^{2}\right\}^{-1}\left\{E_{\mathbf{k}+\mathbf{q}_{j}}^{2}-E_{\mathbf{k}}^{2}\right\}^{-1}\left\{E_{\mathbf{k}+\mathbf{q}_{i}}+E_{\mathbf{k}}\right\}\left\{E_{\mathbf{k}+\mathbf{q}_{j}}+E_{\mathbf{k}}\right\} \\
& =2 \sum_{\mathbf{k}, s} n_{\mathbf{k}, s}\left(\varepsilon_{\mathbf{k}+\mathbf{q}_{i}}-\varepsilon_{\mathbf{k}}\right)^{-1}\left(\varepsilon_{\mathbf{k}+\mathbf{q}_{j}}-\varepsilon_{\mathbf{k}}\right)^{-1} C\left(\mathbf{k} \mid \mathbf{q}_{i}\right) C\left(\mathbf{k} \mid \mathbf{q}_{j}\right)
\end{aligned}
$$

where $\varepsilon_{k}=\hbar^{2} k^{2} / 2 m_{0}$ and the function $C\left(\mathbf{k}, \mathbf{q}_{i}\right)$ is defined by the next relation

$$
C\left(\mathbf{k} \mid \mathbf{q}_{i}\right)=\frac{1}{2}\left\{\left(1+y^{2}[\mathbf{k}+\mathbf{q}]^{2}\right)^{1 / 2}+\left(1+y^{2} k^{2}\right)^{1 / 2}\right\},
$$

and vectors $\mathbf{k}$, and $\mathbf{q}$, and $q$ are measured in $k_{F}$ units. As in the previous section, we use the long-wave approximation, performing the substitution $C\left(\mathbf{k} \mid \mathbf{q}_{i}\right) \Rightarrow\left(1+y^{2} k^{2}\right)^{1 / 2} \equiv C_{k}$. This allows us to use the Feynman identity and to integrate with respect to the $\mathbf{k}$-vector angles and to reduce the $\gamma_{3}\left(\mathbf{q}_{i}, \mathbf{q}_{j}\right)$ calculation to the one-dimensional integral, without any other approximations. In accordance with the Feynman identity

$$
\begin{aligned}
\left(\varepsilon_{\mathbf{k}+\mathbf{q}_{i}}-\varepsilon_{\mathbf{k}}\right)^{-1}\left(\varepsilon_{\mathbf{k}+\mathbf{q}_{j}}-\varepsilon_{\mathbf{k}}\right)^{-1} & =\left(\frac{\hbar^{2}}{2 m_{0}}\right)^{-2} \int_{0}^{1} d x F^{-2}\left(\mathbf{q}_{i}, \mathbf{q}_{i} \mid \mathbf{k}\right), \\
F\left(\mathbf{q}_{i}, \mathbf{q}_{j} \mid \mathbf{k}\right) & =x\left[q_{i}^{2}+2\left(\mathbf{k}, \mathbf{q}_{i}\right)\right]+(1-x)\left[q_{j}^{2}+2\left(\mathbf{k}, \mathbf{q}_{j}\right)\right]=\Omega_{i j}+2\left(\mathbf{k}, \boldsymbol{\rho}_{i j}\right), \\
\Omega_{i j} & \equiv q_{j}^{2}+x\left(q_{i}^{2}-q_{j}^{2}\right) ; \quad \boldsymbol{\rho}_{i j}=x \mathbf{q}_{i}+(1-x) \mathbf{q}_{j} .
\end{aligned}
$$

Now proceed from the sum of the vector $\mathbf{k}$ to the integral form where the integration is performed in a spherical coordinate system in which $O z$ axis coincides with the vector $\boldsymbol{\rho}_{i j}$. As a result of these 
operations we obtain the representation

$$
\begin{aligned}
\gamma_{3}\left(\mathbf{q}_{i}, \mathbf{q}_{j}\right) & =\frac{3 N_{e}}{\left(m_{0} c^{2} y^{2}\right)^{2}} \int_{0}^{1} d k\left\{1+y^{2} k^{2}\right\} f_{i j}(k), \\
f_{i j}(k) & =-\frac{1}{2} \int_{0}^{1} \frac{d x}{\rho_{i j}^{2}-\frac{1}{4 k^{2}} \Omega_{i j}^{2}} .
\end{aligned}
$$

In the explicit form, the square trinomial that appears here is written foolows

$$
\begin{aligned}
\rho_{i j}^{2}-\frac{1}{4 k^{2}} \Omega_{i j}^{2} & =C_{i j} x^{2}+B_{i j} x+A_{i j}, \\
C_{i j} & =\left(\mathbf{q}_{i}-\mathbf{q}_{j}\right)^{2}-\frac{1}{4 k^{2}}\left(q_{i}^{2}-q_{j}^{2}\right)^{2}, \\
B_{i j} & =2\left\{\left(\mathbf{q}_{i}, \mathbf{q}_{j}\right)-q_{j}^{2}\right\}-\frac{1}{2 k^{2}} q_{j}^{2}\left(q_{i}^{2}-q_{j}^{2}\right), \quad A_{i j}=q_{j}^{2}-\frac{1}{4 k^{2}} q_{j}^{4},
\end{aligned}
$$

moreover

$$
\begin{aligned}
& \delta_{i j}=4 A_{i j} C_{i j}-B_{i j}^{2}=\Delta_{i j}-q_{i}^{2} q_{j}^{2}\left(\mathbf{q}_{i}-\mathbf{q}_{j}\right)^{2} k^{-2}=\Delta_{i j}-\frac{q_{1}^{2} q_{2}^{2} q_{3}^{2}}{k^{2}}=\Delta_{i j}\left\{1-\frac{q_{R}^{2}}{k^{2}}\right\}, \\
& \Delta_{i j}=4 q_{i}^{2} q_{j}^{2}\left(1-t_{i j}^{2}\right), \quad q_{R}^{2}=\left(\mathbf{q}_{i}-\mathbf{q}_{j}\right)^{2}\left\{4\left(1-t_{i j}^{2}\right)\right\}^{-1} .
\end{aligned}
$$

Here $q_{R}$ is the radius of the circle circumscribing the triangle constructed on the vectors $\mathbf{q}_{i}, \mathbf{q}_{j},-\mathbf{q}_{i}-\mathbf{q}_{j}$, $t_{i j}$ is a cosine of the angle between the vectors $\mathbf{q}_{i}$ and $\mathbf{q}_{j}$.

Whereas $\Delta_{i j} \geqslant 0$, we get the following result:

$$
\begin{aligned}
& f_{i j}(k)=\left(-\delta_{i j}\right)^{-1 / 2} \ln \left|\frac{R_{i j}+\left(-\delta_{i j}\right)^{-1 / 2}}{R_{i j}-\left(-\delta_{i j}\right)^{-1 / 2}}\right| \quad \text { by } \quad k<q_{R} \\
& f_{i j}(k)=2\left(\delta_{i j}\right)^{-1 / 2} \arctan \left\{\left(\delta_{i j}\right)^{1 / 2} R_{i j}^{-1}\right\} \quad \text { by } \quad k>q_{R} .
\end{aligned}
$$

Here

$$
R_{i j} \equiv R_{i j}(k)=2 A_{i j}+B_{i j}=2\left(\mathbf{q}_{i}, \mathbf{q}_{j}\right)-\frac{1}{2 k^{2}} q_{i}^{2} q_{j}^{2} .
$$

In accordance with the formula (27), the function $\mu_{3}^{0}\left(\mathbf{q}_{1}, \mathbf{q}_{2},-\mathbf{q}_{1}-\mathbf{q}_{2}\right)$ can be written in the form of the one-dimension integral

$$
\mu_{3}^{0}\left(\mathbf{q}_{1}, \mathbf{q}_{2}, \mathbf{q}_{3}\right)=\frac{3 N_{e}}{\left(2 \varepsilon_{F}\right)^{2}} \int_{0}^{1} d k\left(1+y^{2} k^{2}\right) \Phi\left(k \mid q_{1}, q_{2}, q_{3}\right),
$$

where

$$
\Phi\left(k \mid q_{1}, q_{2}, q_{3}\right)=f_{12}(k)+f_{23}(k)+f_{31}(k) .
$$

The sum of terms in the formula (33) can be reduced to a compact form, using the fact that elements $\Delta_{i j}$ and $\delta_{i j}$ are the invariants of this problem:

$$
\Delta_{12}=\Delta_{23}=\Delta_{31}, \quad q_{R}^{2}=\frac{q_{3}^{2}}{4\left(1-t_{12}^{2}\right)}=\frac{q_{2}^{2}}{4\left(1-t_{13}^{2}\right)}=\frac{q_{1}^{2}}{4\left(1-t_{23}^{2}\right)}
$$



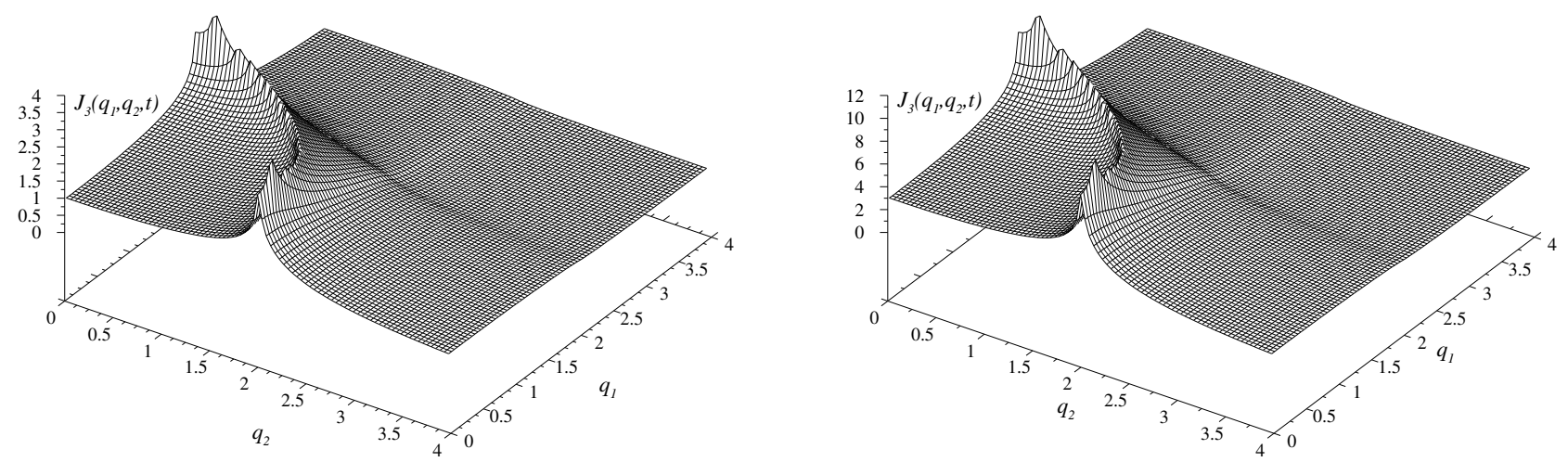

Fig. 4. The function $J_{3}\left(q_{1}, q_{2}, t\right)=\mu_{3}^{0}\left(\mathbf{q}_{1}, \mathbf{q}_{2},-\mathbf{q}_{1}-\mathbf{q}_{2}\right)\left(3 N_{e}\right)^{-1}\left(m_{0} c^{2} y^{2}\right)^{2}$ at $t=0$ and different values of relativistic parameter (from left $y=0$; from right $y=1.0$ ).
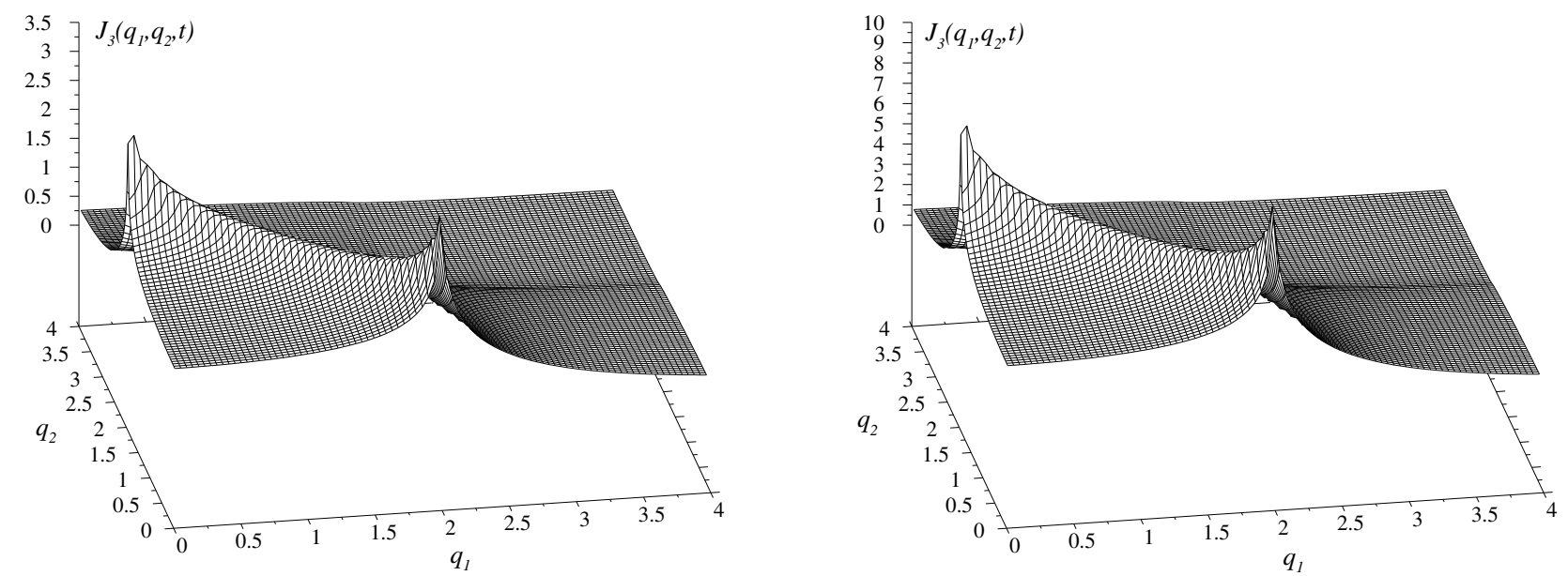

Fig. 5. The function $J_{3}\left(q_{1}, q_{2}, t\right)=\mu_{3}^{0}\left(\mathbf{q}_{1}, \mathbf{q}_{2},-\mathbf{q}_{1}-\mathbf{q}_{2}\right)\left(3 N_{e}\right)^{-1}\left(m_{0} c^{2} y^{2}\right)^{2}$ at $t=0.5$ and different values of relativistic parameter (from left $y=0$; from right $y=1.0$ ).

where $q_{3} \equiv\left|\mathbf{q}_{1}+\mathbf{q}_{2}\right|$. The final result is as follows:

$$
\begin{aligned}
& \Phi\left(k \mid q_{1}, q_{2}, q_{3}\right)=\frac{k}{\gamma_{1}(k)} \cdot \frac{2}{q_{1} q_{2} q_{3}} \ln \left|\frac{1+\gamma_{1}(k) D_{k}}{1-\gamma_{1}(k) D_{k}}\right| \quad \text { by } \quad k<q_{R} ; \\
& \Phi\left(k \mid q_{1}, q_{2}, q_{3}\right)=\frac{k}{\gamma_{2}(k)} \cdot \frac{4}{q_{1} q_{2} q_{3}} \operatorname{arctg}\left\{\gamma_{2}(k) D_{k}\right\} \quad \text { by } \quad k>q_{R} .
\end{aligned}
$$

Here the following notations are used:

$$
\begin{aligned}
\gamma_{1}(k) & =\left[1-\frac{k^{2}}{q_{R}^{2}}\right]^{1 / 2} ; \quad \gamma_{2}(k)=\left[\frac{k^{2}}{q_{R}^{2}}-1\right]^{1 / 2} ; \\
D_{k} & =\frac{q_{1} q_{2} q_{3}}{4 k^{3}}\left(1-\frac{1}{8 k^{2}}\left(q_{1}^{2}+q_{2}^{2}+q_{3}^{2}\right)\right)(P(k))^{-1} \\
P(k) & =1-\frac{q_{1}^{2}+q_{2}^{2}+q_{3}^{2}}{4 k^{2}}+\frac{q_{1}^{4}+q_{2}^{4}+q_{3}^{4}}{32 k^{4}}+\frac{\left(q_{1} q_{2} q_{3}\right)^{2}}{64 k^{6}} .
\end{aligned}
$$

The functions $\Phi\left(k \mid q_{1}, q_{2}, q_{3}\right)$ and $\mu_{3}^{0}\left(\mathbf{q}_{1}, \mathbf{q}_{2},-\mathbf{q}_{1}-\mathbf{q}_{2}\right)$ actually depend on independent variables $q_{1}, q_{2}, t \equiv \cos \left(\mathbf{q}_{1}, \mathbf{q}_{2}\right)$. The dimensionless factor $J_{3}\left(q_{1}, q_{2}, t\right)=\mu_{3}^{0}\left(\mathbf{q}_{1}, \mathbf{q}_{2},-\mathbf{q}_{1}-\mathbf{q}_{2}\right)\left(3 N_{e}\right)^{-1}\left(m_{0} c^{2} y^{2}\right)^{2}$ at the different orientations of vectors $\mathbf{q}_{1}$ and $\mathbf{q}_{2}$ is presented in Fig. 4, 5. 
If in the formula (27) we replace the factor $\left(1+y^{2} k^{2}\right)$ on $\left(1+y^{2}\right)$, we obtain:

$$
\begin{aligned}
\gamma_{3}\left(\mathbf{q}_{i}, \mathbf{q}_{j}\right) & =\frac{3 N_{e}}{\left(m_{0} c^{2} y^{2}\right)^{2}}\left(1+y^{2}\right) \tilde{\gamma_{i j}} \\
\tilde{\gamma_{i j}} & =-2 \int_{0}^{1} \frac{d x}{\rho_{i j}^{2}}\left\{1+\frac{\Omega_{i j}}{4 \rho_{i j}} \ln \left|\frac{2 \rho_{i j}-\Omega_{i j}}{2 \rho_{i j}+\Omega_{i j}}\right|\right\}=\varphi_{i j}-\frac{\delta_{i j}}{\Delta_{i j}} I_{i j}, \quad I_{i j}=\int_{0}^{1} \frac{d x}{\rho_{i j}^{2}-\frac{\Omega_{i j^{2}}}{4}} \\
\varphi_{i j} & =-\frac{2 q_{i}}{\Delta_{i j}}\left\{q_{j}^{2}-\left(\mathbf{q}_{i}, \mathbf{q}_{j}\right)\right\} \ln \left|\frac{1-\frac{1}{2} q_{i}}{1+\frac{1}{2} q_{i}}\right|-\frac{2 q_{j}}{\Delta_{i j}}\left\{q_{i}^{2}-\left(\mathbf{q}_{i}, \mathbf{q}_{j}\right)\right\} \ln \left|\frac{1-\frac{1}{2} q_{j}}{1+\frac{1}{2} q_{j}}\right|
\end{aligned}
$$

Reducing the sum of $\gamma_{3}\left(\mathbf{q}_{1},-\mathbf{q}_{2}\right)+\gamma_{3}\left(\mathbf{q}_{2}, \mathbf{q}_{1}+\mathbf{q}_{2}\right)+\gamma_{3}\left(\mathbf{q}_{1}, \mathbf{q}_{1}+\mathbf{q}_{2}\right)$ to a compact form, we obtain $\mu_{3}^{0}\left(\mathbf{q}_{1}, \mathbf{q}_{2},-\mathbf{q}_{1}-\mathbf{q}_{2}\right)$ the analytical representation which is similar to the introduced in the works [9,10]:

$$
\begin{gathered}
\mu_{3}^{0}\left(\mathbf{q}_{1}, \mathbf{q}_{2},-\mathbf{q}_{1}-\mathbf{q}_{2}\right) \cong \frac{3 N_{e}}{\left(m_{0} c^{2} y^{2}\right)^{2}}\left(1+y^{2}\right) \frac{4 q_{R}^{2}}{q_{1} q_{2} q_{3}}\left\{\sum_{i=1}^{3} \cos \theta_{i} \ln \left|\frac{1+q_{i} / 2}{1-q_{i} / 2}\right|-Y\left(q_{1}, q_{2}, q_{3}\right)\right\} \\
Y\left(q_{1}, q_{2}, q_{3}\right)=\frac{1}{2} \gamma_{2} \ln \left|\frac{1-\gamma_{2} D}{1+\gamma_{2} D}\right| \quad \text { by } \quad q_{R}>1 \\
Y\left(q_{1}, q_{2}, q_{3}\right)=\gamma_{1} \operatorname{arctg}\left[\gamma_{1} D\right] \quad \text { by } \quad q_{R}<1 \\
D=\frac{1}{4} q_{1} q_{2} q_{3}\left[1-\frac{1}{8}\left(q_{1}^{2}+q_{2}^{2}+q_{3}^{2}\right)\right]\left\{1-\frac{1}{4}\left(q_{1}^{2}+q_{2}^{2}+q_{3}^{2}\right)+\frac{1}{32}\left(q_{1}^{4}+q_{2}^{4}+q_{3}^{4}\right)+\frac{1}{64}\left(q_{1} q_{2} q_{3}\right)^{2}\right\}^{-1} \\
\gamma_{1}=\left[\frac{1}{q_{R}^{2}}-1\right]^{1 / 2} ; \quad \gamma_{2}=\left[1-\frac{1}{q_{R}^{2}}\right]^{1 / 2} .
\end{gathered}
$$

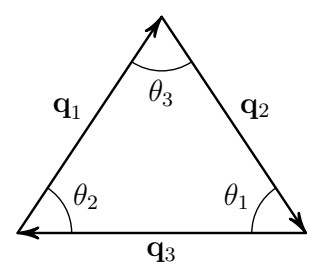

Fig. 6.

Here $q_{3}=\left|\mathbf{q}_{1}+\mathbf{q}_{2}\right|$ and the angle $\theta_{i}$ is on the opposite side of $q_{i}$ triangle is formed by vectors $\mathbf{q}_{1}, \mathbf{q}_{2}, \mathbf{q}_{3}$, as shown in Fig. 6.

One can obtain the exact analytical expression without described above replacement but the result is very cumbersome and we do not give it here.

\section{The local field correction function}

Doing the summation over the frequencies $\nu_{1}$ and $\nu^{*}\left(\nu^{*}\right.$ appears in $\left.\mu_{4}^{0}\left(x,-x, x_{1},-x_{1}\right)\right)$, using the formula (9), we represent the expression (6) in the following form:

$$
\begin{aligned}
G_{i d}(x)= & -V_{q}^{-1}\left\{\mu_{2}^{0}(x,-x)\right\}^{-2} \sum_{s} \sum_{\mathbf{k}_{1}, \mathbf{k}_{2}} n_{\mathbf{k}_{1}, s} n_{\mathbf{k}_{2}, s} \\
& \times\left\{V\left(\mathbf{k}_{1}-\mathbf{k}_{2}\right) f_{\mathbf{k}_{1}, \mathbf{k}_{2}}^{-}(\mathbf{q}, \nu)-V\left(\mathbf{k}_{1}+\mathbf{k}_{2}+\mathbf{q}\right) f_{\mathbf{k}_{1}, \mathbf{k}_{2}}^{+}(\mathbf{q}, \nu)\right\}, \\
f_{\mathbf{k}_{1}, \mathbf{k}_{2}}^{\mp}(\mathbf{q}, \nu)= & \operatorname{Re}\left\{\left[i \nu+E_{\mathbf{k}_{1}}-E_{\mathbf{k}_{1}+\mathbf{q}}\right]^{-1} \mp\left[ \pm i \nu+E_{\mathbf{k}_{2}}-E_{\mathbf{k}_{2}+\mathbf{q}}\right]^{-1}\right\}^{2} .
\end{aligned}
$$

In the asymptotic of small and large values of the wave vector $\mathbf{q}$, the expression (39) coincides with the asymptotic of the local field correction in a non-relativistic theory [13]

$$
G_{i d}(x) \Rightarrow\left\{\begin{array}{l}
\frac{1}{4}\left(q / k_{F}\right)^{2}+\cdots, \quad \nu=0 ; \quad q \ll k_{F} ; \\
\frac{3}{20}\left(q / k_{F}\right)^{2}+\cdots, \quad \nu \gg \varepsilon_{F} ; \quad q \ll k_{F} ; \\
\frac{1}{3}+\cdots, \quad q \gg k_{F} \quad \text { for all } \nu
\end{array}\right.
$$


For the numerical calculation of $G_{i d}(x)$ at absolute zero temperature we use a cylindrical coordinate system for the vectors $\mathbf{k}_{1}, \mathbf{k}_{2}\left(\mathbf{k}_{j}=\left(z_{j}, \rho_{j}, \varphi_{j}\right)\right)$, where $\left(\mathbf{k}_{j}, \mathbf{q}\right)=q z_{j},\left(\mathbf{k}_{1}-\mathbf{k}_{2}\right)^{2}=\rho_{1}^{2}+\rho_{2}^{2}+\left(z_{1}-\right.$ $\left.z_{2}\right)^{2}-2 \rho_{1} \rho_{2} \cos \left(\varphi_{1}-\varphi_{2}\right),\left(\mathbf{k}_{1}, \mathbf{k}_{2}\right)=z_{1} z_{2}+\rho_{1} \rho_{2} \cos \left(\varphi_{1}-\varphi_{2}\right), k_{j}^{2}=z_{j}^{2}+\rho_{j}^{2}$. Doing the integration with respect to variables $\varphi_{1}, \varphi_{2}$, we reduce $G_{i d}(x)$ to the following 4-dimensional integral:

$$
\begin{aligned}
G_{i d}(q, \nu)= & \frac{q^{2} y^{4}}{8} J_{2}^{-2}\left(q y, \tilde{\nu} y^{2}\right) \int_{-1}^{1} d z_{1} \int_{-1}^{1} d z_{2} \int_{0}^{\sqrt{1-z_{1}^{2}}} \rho_{1} d \rho_{1} \int_{0}^{\sqrt{1-z_{2}^{2}}} \rho_{2} d \rho_{2} \times \\
& \times\left\{\frac{f_{q, \nu}^{+}\left(z_{1}, z_{2}, \rho_{1}, \rho_{2}\right)}{W_{+}\left(z_{1}, z_{2}, \rho_{1}, \rho_{2}\right)}-\frac{f_{q, \nu}^{-}\left(z_{1}, z_{2}, \rho_{1}, \rho_{2}\right)}{W_{-}\left(z_{1}, z_{2}, \rho_{1}, \rho_{2}\right)}\right\}
\end{aligned}
$$

where

$$
\begin{aligned}
f_{q, \nu}^{ \pm}\left(z_{1}, z_{2}, \rho_{1}, \rho_{2}\right) & =\left\{\frac{\eta_{1}}{\eta_{1}^{2}+\bar{\nu}^{2}} \pm \frac{\eta_{2}}{\eta_{2}^{2}+\bar{\nu}^{2}}\right\}^{2}-\bar{\nu}^{2}\left\{\frac{1}{\eta_{1}^{2}+\bar{\nu}^{2}}-\frac{1}{\eta_{2}^{2}+\bar{\nu}^{2}}\right\}^{2}, \\
\eta_{i} & =\left[1+y^{2}\left(z_{i}^{2}+\rho_{i}^{2}\right)\right]^{1 / 2}-\left[1+y^{2}\left(\rho_{i}^{2}+\left(z_{i}+q\right)^{2}\right)\right]^{1 / 2}, \\
W_{+}\left(z_{1}, z_{2}, \rho_{1}, \rho_{2}\right) & =\left\{\left(z_{1}+z_{2}+q\right)^{4}+\left(\rho_{1}^{2}-\rho_{2}^{2}\right)^{2}+2\left(\rho_{1}^{2}+\rho_{2}^{2}\right)\left(z_{1}+z_{2}+q\right)^{2}\right\}^{1 / 2}, \\
W_{-}\left(z_{1}, z_{2}, \rho_{1}, \rho_{2}\right) & =\left\{\left(z_{1}-z_{2}\right)^{4}+\left(\rho_{1}^{2}-\rho_{2}^{2}\right)^{2}+2\left(\rho_{1}^{2}+\rho_{2}^{2}\right)\left(z_{1}-z_{2}\right)^{2}\right\}^{1 / 2} .
\end{aligned}
$$

Proceeding to the limit $y \rightarrow 0$ in the formulas (41), (42), we obtain the local field correction function that corresponds to Geldart-Taylor approximation for polarization function in the first order of perturbation theory of non-relativistic model [13]:

$$
\begin{aligned}
G_{i d}^{0}(q, \nu)= & \frac{1}{8}\left\{J_{2}(q, \nu)\right\}^{-2} \int_{-1}^{1} d z_{1} \int_{-1}^{1} d z_{2} \int_{0}^{\sqrt{1-z_{1}^{2}}} \rho_{1} d \rho_{1} \int_{0}^{\sqrt{1-z_{2}^{2}}} \rho_{2} d \rho_{2} \\
& \times\left\{\frac{\varphi_{q, \nu}^{+}\left(z_{1}, z_{2}, \rho_{1}, \rho_{2}\right)}{W_{+}\left(z_{1}, z_{2}, \rho_{1}, \rho_{2}\right)}-\frac{\varphi_{q, \nu}^{-}\left(z_{1}, z_{2}, \rho_{1}, \rho_{2}\right)}{W_{-}\left(z_{1}, z_{2}, \rho_{1}, \rho_{2}\right)}\right\}, \\
\varphi_{q, \nu}^{ \pm}\left(z_{1}, z_{2}, \rho_{1}, \rho_{2}\right)= & \left\{\frac{z_{1}+q / 2}{\left(z_{1}+q / 2\right)^{2}+u^{2}} \pm \frac{z_{2}+q / 2}{\left(z_{2}+q / 2\right)^{2}+u^{2}}\right\}^{2} \\
& -u^{2}\left\{\frac{1}{\left(z_{1}+q / 2\right)^{2}+u^{2}}-\frac{1}{\left(z_{2}+q / 2\right)^{2}+u^{2}}\right\}^{2}, \\
u= & \nu\left[\frac{\hbar^{2} k_{F}^{2}}{m} q\right]^{-1}, \quad q \equiv|\mathbf{q}| k_{F}^{-1},
\end{aligned}
$$

where the functions $W_{ \pm}\left(z_{1}, z_{2}, \rho_{1}, \rho_{2}\right)$ is determined by the formulas $(42)$, and $J_{2}(q, \nu)$ - by the formula (12). In this approximation, the local field correction is obtained for the first time in the paper [5]. Since $\varphi_{q, \nu}^{ \pm}\left(z_{1}, z_{2}, \rho_{1}, \rho_{2}\right)$ does not depend on the variables $\rho_{1}, \rho_{2}$, then integrating with respect to these variables is performed in the analytical form and $G_{i d}(q, \nu)$ is reduced to the calculation of the twodimensional integral with respect to the variables $z_{1}, z_{2}$ with the help of numerical method. For the comparison $G_{i d}(q, \nu)$ with the local field correction in a non-relativistic theory, in the formulas (41), (42) the variables $q=|\mathbf{q}| k_{F}^{-1}$ and $\bar{\nu}=\tilde{\nu} y^{2}, \tilde{\nu}=\nu\left(\hbar^{2} k_{F}^{2} / m\right)^{-1}$ are used.

Fig. 7 shows the dynamic local field correction function calculated by the formula (41) with $\tilde{\nu}=0.01$, which is very close to the static limit. As it shown in the figure, the asymptotic behavior $G_{i d}(q, \nu)$ for small and large values of the wave vector almost do not depend on the relativistic parameter and its asymptotic coincides with the asymptotic of the function $G_{i d}^{0}(q, \nu)$. The deviation of $G_{i d}(q, \nu)$ from $G_{i d}^{0}(q, \nu)$ is significant in the domain of its maximum value, which decreases monotonically with the 


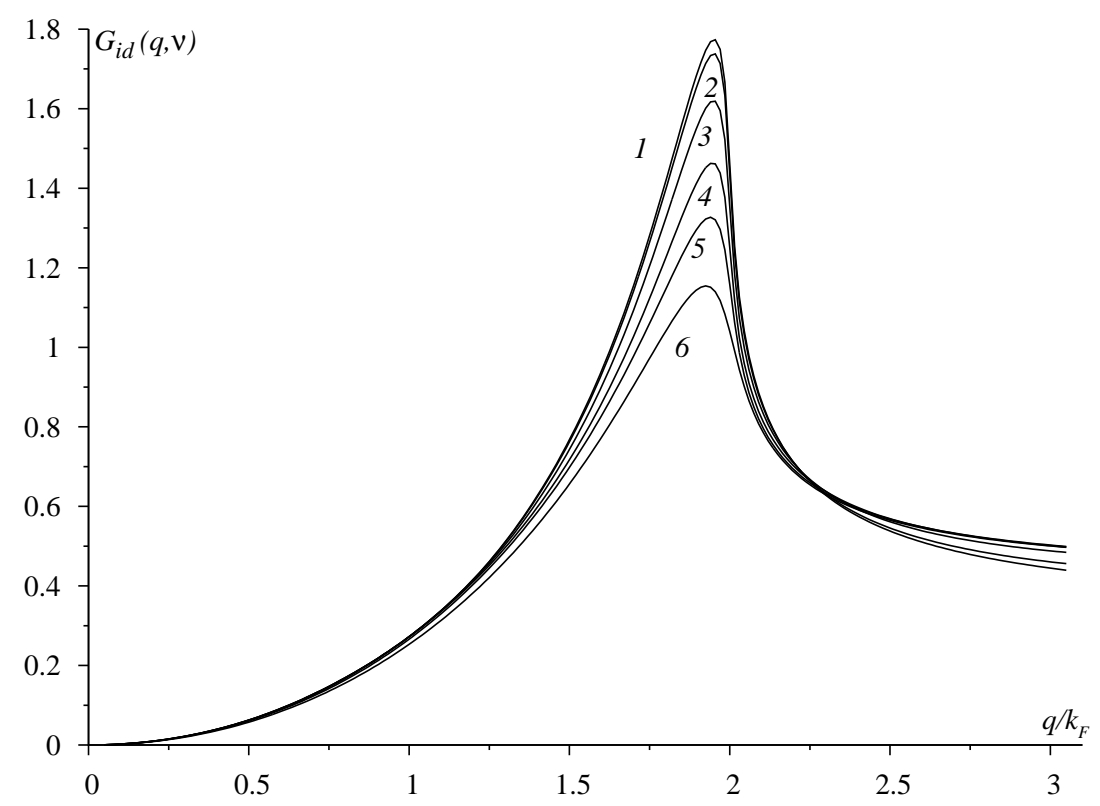

Fig. 7. Dependence of the local field correction function $G_{i d}(q, \nu)$ on the wave vector $\mathbf{q}$ and relativistic parameter $y$ for the frequency $\nu=0.01 m_{0} c^{2} y^{2}$ (curve $1-y=0.05$; $2-y=0.2 ; 3-y=0.5 ; 4-y=1 ; 5-y=2 ; 6-y=5)$.

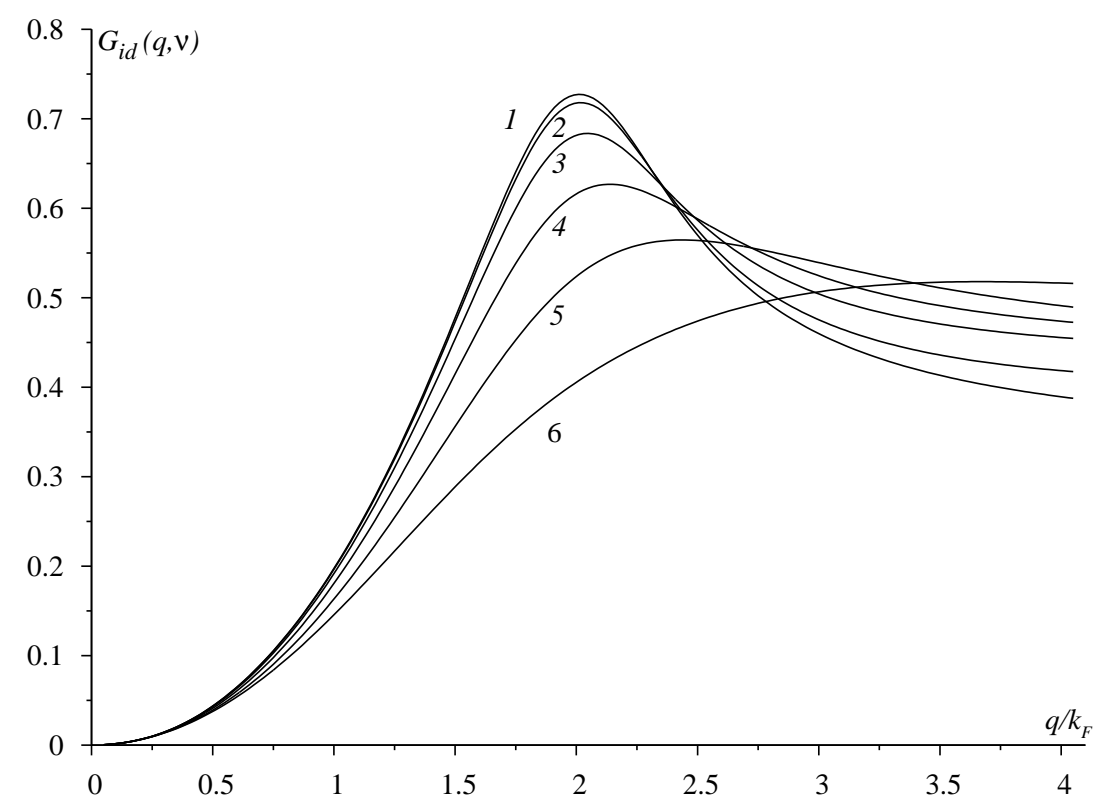

Fig. 8. Dependence of the local field correction function $G_{i d}(q, \nu)$ on the wave vector $\mathbf{q}$ and relativistic parameter $y$ for the frequency $\nu=0.5 m_{0} c^{2} y^{2}$ (curve $1-y=0.05$;

$$
2-y=0.2 ; 3-y=0.5 ; 4-y=1 ; 5-y=2 ; 6-y=5) \text {. }
$$

increase of relativistic parameter. Fig. 8 shows the dependence of $G_{i d}(q, \nu)$ on the wave vector for a sufficiently large value of the frequency $(\tilde{\nu}=0.5)$. In this case, the dependence character on the wave vector corresponds to the behavior of non-relativistic correction, but the maximum height is significantly reduced with the increase of relativistic parameter due to the frequency renormalization $\left(\nu_{*}=\tilde{\nu} y^{2}\right)$ for $y \gtrsim 1$. This remark relates also to a shift of the maximum value to the large wave vector domain. 


\section{Conclusions}

The correlation functions of the ideal degenerate electron gas model in the momentum-frequency representation $\mu_{n}^{0}\left(x_{1}, \cdots, x_{n}\right)$ are the universal characteristics of this model, which at the low temperatures depend only on the dimensionless relativistic parameter which in its turn is determined by the particles concentration. The momentum-frequency dependence of correlation functions for the different values of relativistic parameter is similar. For the small values of this parameter, the functions $\mu_{n}^{0}\left(x_{1}, \cdots, x_{n}\right)$ are well known. In the internal structure theory of degenerated dwarfs the necessity of correlation functions calculation with arbitrary relativistic parameter value arises. As it follows from our calculations in the high density case the role of high order correlation effects is decreasing: $\mu_{2}^{0}\left(x_{1},-x_{1}\right) \sim 1 / y, \mu_{3}^{0}\left(x_{1}, x_{2},-x_{1}-x_{2}\right) \sim 1 / y^{2}, \mu_{4}^{0}\left(x_{1},-x_{1}, x_{2},-x_{2}\right) \sim 1 / y^{3}$ etc., which corresponds to a weak non-ideality of this model. This is the reason to use the local field correction function in the lowest approximation based on the functions $\mu_{4}^{0}\left(x_{1},-x_{1}, x_{2},-x_{2}\right)$. We have investigated the features of two- and three-particle correlation functions in the wide domain of relativistic parameter, obtained the exact expression for the static two-particle correlation function, approximate expressions for the three-particle correlation function for the first time, studied the local field correction of interacting relativistic electron gas, which is the basis for calculating the energy and structural characteristics of degenerated dwarfs.

[1] Chandrasekhar S. The maximum mass of ideal white dwarfs. Astrophys. Journ. 74, 81 (1931).

[2] Chandrasekhar S. Stellar configurations with degenerate cores' (second paper). Mon. Not. Roy. Astron. Soc. 95, 676 (1935).

[3] Salpeter E. Energy and pressure of a zero-temperature plasma. Astrophys. Journ. 134, 669 (1961).

[4] Vavrukh M., Krokhmalskii T. Reference system approach in the electron liquid theory. I. General relations. Phys. Stat. Sol. (b), 168, n. 2, 519 (1991).

[5] Vavrukh M., Krokhmalskii T. Reference system approach in the electron liquid theory. II. Ground state characteristics in the medium density region. Phys. Stat. Sol. (b), 169, 451 (1992).

[6] Vavrukh M., Solovyan V., Vavrukh N. Reference system approach in the electron liquid theory. III. Dynamic function of the local-field correction. Phys. Stat. Sol. (b), 177, 361 (1993).

[7] Vavrukh M., Vavrukh N. Generalization of the local-field concept in the theory of Fermi liquid. Low Temp. Phys. 22, n. 9, 767 (1996).

[8] Gell-Mann M., Brueckner K. Correlation energy of an electron gas at high density. Phys. Rev. 106, 364 (1957).

[9] Brovman E., Kagan Yu. Singularities of multitail ring diagrams for fermi systems. Zh. Eksp. Teor. Fiz. 63 36, n. 5, 1937 (1972).

[10] Lloyd P., Sholl C. A structure expansion of the cohesive energy of simple metals in the effective Hamiltonian approximation. J. Phys. C, 1, n. 2, 1620 (1968).

[11] Hwa R., Teplitz V. Homology and Feynman integrals. New York, Amsterdam, (1968). 223 p.

[12] Vavrukh M., Tyshko N., Smerechynskyj S. Interparticle interactions, general relativity effects, and critical parameters of white dwarfs. Mathematical modeling and computing. 1, n.2, 264-283 (2014).

[13] Geldart D. J. W., Taylor R. Wave-number dependence of the static screening function of an interacting electron gas. Can. J. Phys. 48, n. 2, 155 (1970). 


\title{
Кореляційні функції виродженого релятивістського електронного газу високої густини
}

\author{
Ваврух М. В. ${ }^{1}$, Дзіковський Д. В. ${ }^{1}$, Солов'ян В. Б. ${ }^{2}$, Тишко Н. Л. ${ }^{1}$ \\ 1 Лъвівсъкий національний університет імені Івана Франка \\ вул. Кирила і Мефодія, 8, 79005 Львів, Україна \\ ${ }^{2}$ Інститут фізики конденсованих систем НАН України \\ вул. Свєнціцького, 1, 79011, Львів, Украӥна
}

\begin{abstract}
Досліджено і розраховано дво- і тричастинкові кореляційні функції моделі виродженого релятивістського однорідного електронного газу з кулонівськими взаємодіями за $T=0 K$ в імпульсно-частотному зображенні у наближенні локального поля. Цих функцій достатньо для коректного розрахунку рівняння стану електрон-ядерної моделі за густин, що відповідають виродженим карликам.
\end{abstract}

Ключові слова: базисний підхід, п-частинкові кореляиійні функцій, поправка на локальне поле.

2000 MSC: 82B10, 82D10

УДК: $537.31 .311,53.01$ 